\title{
Siddha herbs for obesity
}

\author{
J. Raamachandran ${ }^{1} \&$ T. Venkatasubramaniam ${ }^{2}$ \\ ${ }^{1}$ Murugan Siddha Marunthakam, Murugan Illam, India \\ 2 "Rekha Cuisine”, Old Basing, Basingstoke, UK
}

\begin{abstract}
The Siddha medicinal system developed by Tamil Siddhars is the oldest medicinal system in India. It has developed medicines in a scientific way. In today's world obesity occupies a significant place since many people are affected by it. Even though obesity is not a new phenomenon, yet, today it has attained such an epidemic proportion to be rechristened as "globesity". The causes for obesity may be genetics, food habits, sedentary life-style and hormonal changes. The Siddha medicinal system developed by Tamil Siddhars deals with this problem according to the root-cause for obesity. Several herbs are in use to control obesity. Some of them are used as part of food and others are used as medicines. The Tamil Siddhars were the first to give the concept of "food is medicine". This paper discusses various herbs and the way they are used.

Keywords: Siddha medicine, genetic problem, sedentary life, hormone imbalance, Andrographis paniculata, Zingiber officinale, Dolichos biflorus, Erthrina Varigata, thermo genesis, BMI, $L D L$.
\end{abstract}

\section{Introduction}

Obesity, once considered a sign from the affluence, has now become a major source of nuisance both aesthetically and from the heath-point of view. Like diabetes which may trigger other diseases, obesity and over-weight are also a cause for many chronic diseases including diabetes, cardiovascular diseases, cancer, etc. Once considered a problem for people in the higher income group, overweight and obesity are now on the rise even among the middle income groups especially in the urban settings. According to a recent survey (OECD Health Data 2005) nearly $30 \%$ of Americans and $23 \%$ of the UK population is obese. The percentage increases almost in all countries causing concern to health authorities. It is necessary to tackle this problem of obesity as it is the root cause 
for several non-communicable diseases, principally cardiovascular diseases, diabetes, chronic respiratory diseases and the like. A study in the Lancet predicted that by 2030 nearly $70 \%$ of all deaths will be from non-communicable diseases.

Obesity and overweight are defined as abnormal or excessive fat accumulation in the body that poses a risk to health. As of now, a crude way of indicating obesity is the body mass index (BMI). The BMI is calculated by dividing a person's weight in kilogram by the square of his or her height in meter $\left(\mathrm{kg} / \mathrm{m}^{2}\right)$. If the BMI is greater than 25 but less than 30 then he or she is overweight. However, if the BMI is $>30$ then the person is considered obese.

\subsection{Causes of obesity}

One obvious reason for obesity is the input and output ratio, that is, the balance between calorie intake and energy spending. If a person eats more calories than he/she spends (metabolizes) then the person's body will store the excess energy as fat which when accumulates over the years will result on overweight and to obese conditions. This type of situation is due to inadequate physical activity. However there are many other factors that contribute to obesity. Some of them are:

\section{$>$ Over-eating}

Some people tend to eat more than necessary and especially food with fat content. The diet may not be a balanced one. According to Siddha philosophy, "food is medicine". One can avoid falling sick by properly eating. To this end the Siddhars gave the concept of "aru suvai unavu", that is, diet with six tastes. These are astringent, sour, sweet, salt, bitter and hot. A diet with combination of food with these tastes is equivalent to modern "balanced food" [1]. In modern days it is also becoming a fashion to go after "fast food", fried food etc. which contribute to not only obesity but also other health problems. A non-balanced diet is as good as malnutrition though tend to cause over-weight.

\section{$>$ Genetics}

Genetics also plays a major role in causing obesity in children especially if both parents are obese.

\section{$>$ Hormone problem}

Thyroid gland dysfunction is another cause for obesity as the hormone secreted by it essentially regulates the body's metabolism which determines the rate at which a person can burn calories and thus has the ultimate effect on one's weight. With reference to obesity we talk about underactive thyroid, a disorder known as "hypothyroidism". That is, thyroid hormone secretion is not enough to maintain the metabolic activity.

In the Siddha medicinal system there are various herbs used to tackle obesity according to root cause for the same. Some of them can be used as part of food and others will be used only as medicine. We will discuss these one by one. 


\section{Herbs used as food}

\subsection{Murraya Koenigii ("kariveppilai” in Tamil)}

Commonly known as "curry leaves", one cannot find a single house in Tamil naadu and elsewhere without the leaves of Murraya koenigii in its kitchen. It is also a common sight to see the growing of this plant in many homes especially in the village side. However, every grocery shop will sell these leaves as it is widely used almost in most of the preparation like saambaar, rasam (types of Tamil cuisine) and other dishes. While it is customary to add few leaves of Murraya koenigii in the above preparation it is also used alone to cure certain physical ailments. Obesity is one such ailment for which Murraya Koenigii is used with success. Murraya Koenigii is especially useful in cases where obesity is caused by improper digestion of food and over eating. Murraya Koenigii is very good in burning calories which helps in controlling one's weight.

The plant is medium sized tree that grows up to 3 to 4 meters. The leaves are imparipinnate and leaflets rhomboid. The flowers are white and in terminal cyme, each bearing 60 to 90 flowers and the average diameter of a fully opened flower is around $1 \mathrm{~cm}$ (Fig. 1).

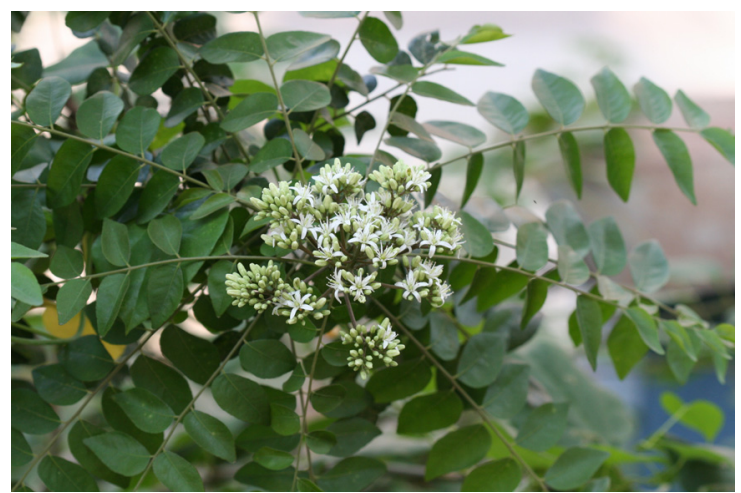

Figure 1: $\quad$ Murraya Koenigii.

The smooth and shining leaves of Murraya Koenigii may resemble that of the neem tree. However the leaves do not have teeth like that of neem. While the leaves, ribs and bark are medicinally useful, only the leaves are used in cuisines. The phytochemicals present in the leaves are: mahanimbine, girinimbine, isomahanimbine koenimbine, koengicine etc. [1]. The method of using Murraya Koenigii as part of food is given below.

Recipe 1: Dry the leaves of Murraya koenigii in shade and powder them. Take about 200 grams of the powder. To this add 20 grams each of pepper, cumin seeds and dried ginger, all in the powdered form. Add salt to taste. Mix them well. Mix a table spoon of this powder with cooked rice (cooked rice is called "saatham" in Tamil) and eat at the first serving. This is good for diabetes, 
indigestion and constipation. It should be mentioned that about 1 gram of melted ghee should be added to the above rice before eating. This way full benefit can be obtained. It is also to be mentioned that diabetic people must use only brown rice ("kai kuththal arisi" - that is hand polished rice in Tamil).

Recipe 2: Kariveppilai saatham: This is a common dish normally made in houses and also during functions like marriages. It is prepared thus: Take fresh leaves of Murraya koenigii 100grams; dried red chilli, 10 grams, tomato and onion 1 each of normal size; mustard and black gram each 1 tea-spoon and sesame or olive oil 2 table spoons.

Method: Pour oil in a saucepan and when it is hot put mustard and blackgram. Allow the mustard to splatter. This process is known as "thaaliththal" in Tamil. Then add finely chopped Murraya leaves, tomato and onion. Fry them well. Then grind them to a paste form. Add salt to taste. This is called "kariveppilai thuvaiyal". Take enough of the thuvaiyal and mix with saatham. Normally 5 grams of thuvaiyal can be added to 50 grams of saatham. Eating this regularly as a first thing during lunch will control obesity and diabetes and cure it eventually. This will also cure indigestion and remove tastelessness. This will increase insulin secretion in the body.

There have been several clinical studies that show the anti-obesity effect of Murraya Koenigii. The anti-obesity and lipid lowering effects of Murraya koenigii was studied by Birari et al. [2]. They gave the dichloromethane (MKD) and ethyl acetate (MKE) extracts of Murraya koenigii leaves orally at a dose of $300 \mathrm{mg}$ $/ \mathrm{kg} /$ day to the high fat diet induced obese rats and found significant reduction in the body weight gain. The authors concluded that the phytochemical mahanimbine present in Murraya koenigii is responsible for controlling obesity. While the study proves what the Siddha medicinal system predicted more than 2000 years ago, however, the Siddha system attributes such control to stomachic property ("pasith thee thoondi" in Tamil) of Murraya koenigii.

The first author has advised this herb for many people especially to those who are obese genetically. Incidentally such a food will also control diabetes and diabetes related obesity. The result is very satisfactory as seen in Table.1. (Note: In Table 1, (2: 61\%) indicates that for $61 \%$ of the people treated the BMI index number was reduced by 2 from the level before medication started. In a similar way other readings are to be interpreted. All the readings are from the personal clinical observation of the first author and taken over the past 15 years. The results are based on 3 to 6 months' study).

\subsection{Zingiber officinale ("Inji”" in Tamil)}

The plant Zingiber officinale - more commonly called ginger - is widely known and used not only as medicine but also as part of cuisine. There is no home in Tamil nadu without a piece of ginger. The plant is generally 60 to $180 \mathrm{~cm}$. high with smooth and green leaves. The leaf looks like that of miniaturized plantain leaf. Its flowers are small and the pedicles short. The most useful part is its root which has pleasant aromatic odour. It is brownish externally and yellow internally, (Fig. 2). 


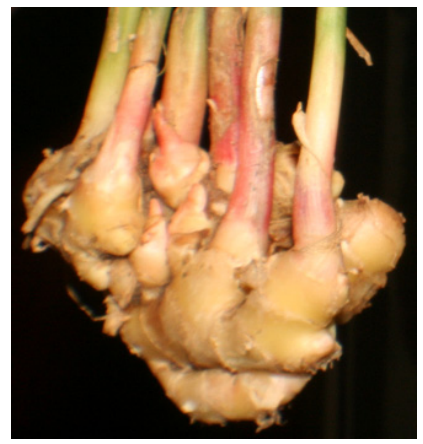

Figure 2: $\quad$ Zingiber officinale.

While fresh ginger is called "inji" in Tamil, the dried one is called "chukku" and both of them are used in medicines. Ginger for its stimulant and carminative properties is used in tooth-ache, gout, rheumatism of the jaws and relaxed uvula. Its decoction is given for colic pain, tympanites, vomiting etc. Ginger boosts thermo genesis, or calorie burning, allowing the body's tissues to use more energy than normal thus helping one to lose weight $[3,4]$.

The phytochemicals present in Zingiber officinale are diarylheptanoids, ginger diol, shogaol and related compounds, essential oil. The dominant sesquiterpene is zinginerene. The less predominants are: cis-sesquisabinene hydrate, zingibernol, ar-curcumine, $\beta$-bisabolene, $\alpha$-selinene, $\beta$-elemente, $\beta$ sesquiphellandrene, zingiberenol. From Siddha medicine point of view Zingiber officinale is stomachic, digestive and carminative. While a piece of Zingiber officinale is normally added to many dishes made in the home, Zingiber officinale is used to control weight in the following way.

Method 1: Crush a piece of Zingiber officinale and take two table spoon of the juice. To this add equal amount of honey and 100 millilitres of warm water. Mix them well and drink on empty stomach. Continued use will reduce one's weight due to its thermo genesis property.

Method 2: Take a small piece of Zingiber officinale and a few pieces of seasalt. Munch them together and then drink warm water. Do this just half-an-hour before lunch or dinner. This will improve digestion and is good for those who have poor digestion and appetite.

The modern analysis indicates that the compounds gingerol and shogaol increase the metabolic rate and thus help to "burn off" excessive fat. They also help to suppress the absorption of calorie-dense dietary fats from the intestines. For these reasons regular intake of ginger should aid in countering excessive weight gain and obesity. The effect of this is quite dramatic, see Table 1.

\subsection{Dolichos Biflorus ("kollu" in Tamil)}

It is more commonly known as horse gram as it was used widely to feed horses. However, in Tamil nadu it is used in Tamil dishes, including kollu porial, kollu 
avial, kollu sambar, and kollu rasam. In traditional Siddha cuisine, horse gram is considered a food with medicinal qualities, (Fig. 3). This pulse is a demulcent in calculus affections, cough etc. It is also used to reduce corpulence. There is even a proverb which says: "iLaiththavanukku ellu; kozhuththavanuuku kollu". That is, give sesame seeds to emaciated and horse gram to corpulent. While the food with sesame seeds will make an emaciated person gaining weight, so also the use of horse gram will make a corpulent to shed of weight. The seeds of Dolichos Biflorus contain demethylhomopterocarpin and some vitamins.

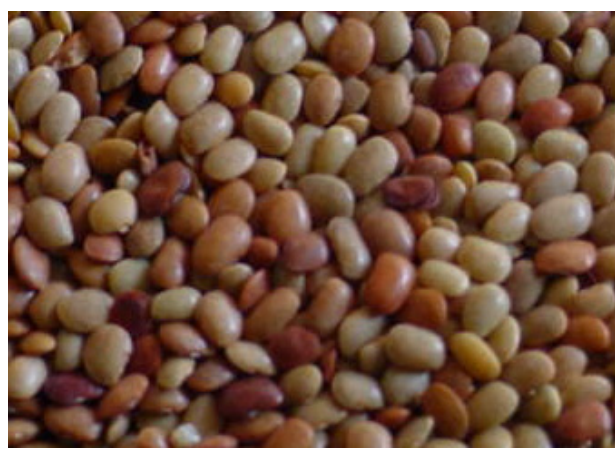

Figure 3: Dolichos biflorus seeds.

Method 1. Soak a tablespoon of Dolichos Biflorus seeds in water overnight. In the morning drink the water and pressure cook the seeds along with a little salt. The cooked seeds can be used to mix with rice and eaten. Continued use will not only shed one's weight but will reduce LDL (Low Density Lipoproteins also known as "bad" cholesterol) level.

Method 2. It is common to make what is known as "kollu podi" to be mixed with steamed rice for eating. This will not only reduce BMI but will also reduce LDL level.

It is made thus:

Take 100 grams of Horse gram seeds; 10 numbers (or 10 grams) of red or dried chillies; half a gram of fried Asafoetida powder and salt to taste. Now, fry horse gram and red chillies without adding any oil. When they cool down add Asafoetida and salt and powder them. This powder can be used to mix with steamed rice with some sesame oil and eaten at a first serving. Repeat 3 times a week.

These recipes are quite effective in cases of genetically obese people. It has also been shown that Dolichos biflorus decreases the level of thiobarbituric acid reactive substance indicating the antioxidant potential of the Dolichos biflorus $[5,6]$ (see Table 1). 
Table 1: $\quad$ BMI reduction values.

\begin{tabular}{|c|c|c|c|}
\hline \multirow{2}{*}{ Herb } & Male & \multicolumn{2}{|c|}{ Female } \\
\cline { 2 - 4 } & Age: $18-52$ years & Age: $18-45$ & Age: $>45$ \\
\hline Murraya Koenigii & $2: 61 \%$ & $2: 64 \%$ & $1: 10 \%$ \\
& $3: 32 \%$ & $3: 30 \%$ & $\ldots$ \\
& $4: 07 \%$ & $4: 6 \%$ & $\ldots$ \\
\hline Ginger & $2: 74 \%$ & $2: 76 \%$ & $1: 23 \%$ \\
& $3: 21 \%$ & $3: 20 \%$ & $\ldots$ \\
& $4: 5 \%$ & $4: 4 \%$ & $\ldots$ \\
\hline Dolichos biflorus & $2: 76 \%$ & $2: 68 \%$ & $1: 18 \%$ \\
& $3: 22 \%$ & $3: 29 \%$ & $\ldots$ \\
& $4: 02 \%$ & $4: 02 \%$ & $\ldots$ \\
\hline Andrographis Paniculata & $2: 65 \%$ & $2: 89 \%$ & $1: 23 \%$ \\
& $3: 28 \%$ & $3: 7 \%$ & $2: 02 \%$ \\
& $4: 07 \%$ & $4: 04 \%$ & $\ldots$ \\
\hline
\end{tabular}

\section{Herbs for hormonal problems}

\subsection{Andrographis paniculata}

Andrographis paniculata is a herb widely used in Siddha medicine for centuries. This is known as ground neem and is very widely used by Tamils for various ailments. Its stem is dark green, 30 to $100 \mathrm{~cm}$ in height, 2 to $6 \mathrm{~mm}$ in diameter, quadrangular with longitudinal furrows and wings on the angles of the younger parts, slightly enlarged at the nodes. Its leaves are glabrous, up to $8 \mathrm{~cm}$ long and $2.5 \mathrm{~cm}$ broad, lanceolate and pinnate. Its flowers are small, in lax spreading axillary and terminal racemes or panicles. The flowers are white with rosepurple spots on the petals. The capsules are linear-oblong, acute at both ends, 1.9 $\mathrm{cm}$ by $0.3 \mathrm{~cm}$ with numerous seeds that are sub quadrate and yellowish brown in colour. The whole plant is bitter in taste. The dried stems are sold in market, (Fig. 4).

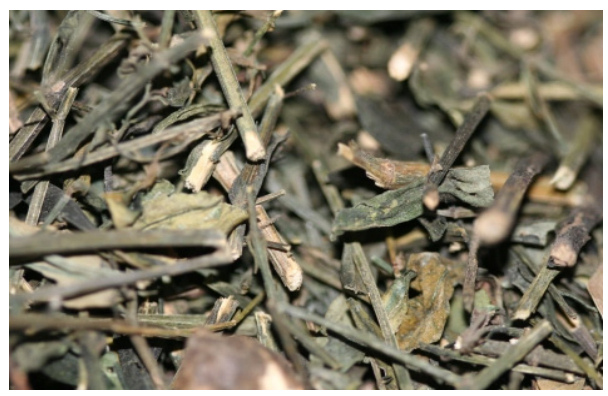

Figure 4: $\quad$ Root of Andrographis paniculata.

The plant is much valued for its stomachic and tonic properties. Mixed with other drugs it is successfully used in general debility and convalescence after fevers. The powder of the stem is quite useful in hypothyroidism which causes 
obesity. In fact, Andrographis paniculata has positive effect on one's immune system.

Half a gram of the powder along with warm water taken on empty stomach for nearly three months restores the function of thyroid.

\section{Menstrual problems}

On close scrutiny of Table 1, one may find that most of the above mentioned herbs did not give satisfactory results in case of women older than 48 years. On examination it as found that most of them were in the menopause stage and some of them even reached menopause. For such women Siddha medicine advises using another herb in conjunction with one or other herbs to be decided case by case. The following herb is for such women.

\subsection{Erythrina variegata ("kaliyaana murukkan" in Tamil)}

The Erythrina variegata is a medium sized tree which grows about 3 to 10 meters in height. The tree has lot of thorns in it so that it is normally grown as hedge plant. Its legume has 6 to 8 seeds. The flower is very attractive and red in colour, (Fig. 5). The fresh bark has a smooth grey colour with unpleasant smell but not very bitter in taste. The bark is used medicinally to reduce bile and it is also febrifuge and anthelmintic. The bark is also used as collyrium in opthalmia. The leaves are normally used not only as medicines but also as part of food. Its medicinal properties are many. The leaves are normally applied externally to cure venereal buboes and to relieve pain in the joints. There is another variety of this tree yielding white flowers and is more useful in medicines. The leaves of this plant are especially used to cure many problems associated with menstrual disorders. The phytochemicals content of this plant are erythratine, ferulic and caffeic acids etc. Alkaloids like erysotine, erythratidine, 11-hydroxy-epierythratidine, epierythratidine etc. isoflavonoids, seadenone, erycristagallin erythrabysin II, phaseollin etc. and fixed oil. The efficacy of Erythrina variegata in reducing cholesterol level was analysed by Balamurugan and Shantha [7].

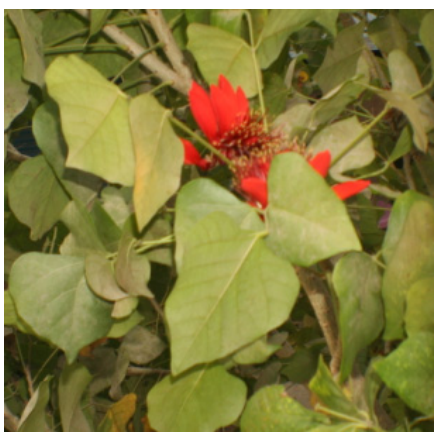

Figure 5: $\quad$ Erythrina variegata. 
Method: Extract the juice from the leaves of Erythrina variegata and drink 20 millilitres at a time for three months. This will not only reduce weight but will also remove stomach pain that normally occurs during menstrual period.

The result of such administration is shown in Table.2. The first row gives the result for those whose obesity is due to digestive problems. The second row gives the result for those whose obesity is due to genetic cause. Menstrual problem is common to both. The results are obtained by the first author after 6 months of continued use of these medicines. As before (1: 78\%) indicates that the BMI number is reduced by 1 for $78 \%$ of the persons treated and so on.

Table 2: $\quad$ BMI reduction in females during and beyond menopause.

\begin{tabular}{|c|c|}
\hline Herb & Age: $>45$ \\
\hline Erythrina variegata + Ginger & $1: 78 \%$ \\
& $2: 64 \%$ \\
\hline Erythrina variegata + Dolichos & $1: 68 \%$ \\
biflorus & $2: 54 \%$ \\
\hline
\end{tabular}

\section{Conclusion}

The oldest medicinal system of India, namely the Siddha medicinal system has several formulae to tackle obesity. Some of the herbs used in the clinics are reported in this paper. Most of them are administered to obese and overweight people and their BMI are monitored over a period to prove the efficacy of Siddha medicines for such problems. It is to be noted that Siddha medicinal system did not simply prescribe herbs for obesity. It goes to the root of obesity, that is, whether obesity is due to genetic reasons or sedentary life or hormonal problem or menstrual problem and then prescribes medicine accordingly. The herbs mentioned in this paper are only a few, but there are several others which are in use for different combination of diseases. For example, if an obese person is also suffering due to blood pressure then Withania somnifera ("amukkaravu" in Tamil) is prescribed and so on. It is hoped that obese community at large will make use of such Siddha medicines to shed off their excessive flesh, the only thing that one will lose using Siddha medicines!

\section{References}

[1] Raamachandran, J., Herbs of Siddha Medicines-The First 3D book on herbs, Murugan Pathippakam, Chennai 600087, 2008

[2] Birari R, Javia V, Bhutani KK., Antiobesity and lipid lowering effects of Murraya koenigii (L.) Spreng leaves extracts and mahanimbine on high fat diet induced obese rats. Fitoterapia. 2010 Dec; 81(8):1129-33

[3] Goyal, R.K., Kadnur, S.V. Beneficial effects of Zingiber officinale on gold thioglucose induced obesity. Fitoterapia 77, (2006) 160-163 
[4] Jeyakumar SM, Nalini N, Venugopal P Menon. Antioxidant activity of ginger (zingiber officinale Rose) in rats fed a high fat diet. Med Sci Res 1999; 27:341-4

[5] Ganga Raju and Rama Raju., Anti-adipogenic compositions containing piper betle and Dolichos biflorus, United States Patent Application 20100203117, 2010

[6] Kottai Muthu A, Sethupathy S, Manavalan R, Karar PK. Hypolipidemic effect of methanolic extract of Dolichos biflorus Linn in high fat diet fed rats. Indian J Exp Biol 2005; 43:522-5

[7] Balamurugan, G and A Shantha., Effect of Erythrina variegata seed extract on hyperlipidemia elicited by high-fat diet in wistar rats, J. of Pharmacy and Bio-Allied Sciences, Year : 2010, Volume : 2, Issue : 4, Page : 350-355 